\title{
Robotic thymectomy for myasthenia gravis surgical techniques and outcomes
}

\author{
Beebarg Raza ${ }^{1}$, Ankit Dhamija ${ }^{2}$, Ghulam Abbas ${ }^{2}$, Alper Toker ${ }^{2}$ \\ ${ }^{1}$ St. George University, Grenada, USA; ${ }^{2}$ Department of Cardiovascular and Thoracic Surgery, West Virginia University, Morgantown, WV, USA \\ Contributions: (I) Conception and design: A Toker, B Raza; (II) Administrative support: G Abbas; (III) Provision of study materials or patients: A \\ Toker, A Dhamija; (IV) Collection and assembly of data: B Raza; (V) Data analysis and interpretation: A Toker, B Raza; (VI) Manuscript writing: All \\ authors; (VII) Final approval of manuscript: All authors. \\ Correspondence to: Alper Toker, MD. West Virginia University School of Medicine, 1 Medical Center Drive, Morgantown, WV 26505-8059, USA. \\ Email: alper.toker@hsc.wvu.edu.
}

\begin{abstract}
Myasthenia gravis (MG) is an autoimmune disorder in which antibodies are produced against post-synaptic acetylcholine receptors, thereby causing impairment of neuromuscular transmission. Diagnosis of MG is confirmed with the AChR antibody test and via an Electromyography. Although medical treatment with acetylcholinesterase inhibitors remains the main treatment of $\mathrm{MG}$, in recent years thymectomy has become an integral part of the treatment algorithm. Numerous factors such as the Patient's age, presence of AChR antibodies, or MuSK antibody, the severity of disease affect the decision of preforming the thymectomy. Historically thymectomy was preformed via sternotomy associated with significant morbidity. Advancement in the minimally invasive approaches to thymic resection has led to more acceptance of thymectomy in the management of MG. Among these approaches, robotic thymectomy is gaining popularity across the globe due to the unique advantages of the robotic platform like $3 \mathrm{D}$ visibility, enhanced dexterity, and wrist like articulating movements of instruments. This has led to less post-operative pain and morbidity; faster recovery and shorter hospital stay. Successful treatment of MG requires a multi-modality approach, which has led to the formation of MG teams in most academic centers, comprising of a specialist neurologist, intensivist, and thoracic surgeon. In this article, we describe the techniques and outcomes of the robotic thymectomy for MG.
\end{abstract}

Keywords: Myasthenia gravis (MG); thymectomy; robotic

Submitted Feb 03, 2020. Accepted for publication Sep 10, 2020.

doi: $10.21037 /$ jtd-2019-rts-10

View this article at: http://dx.doi.org/10.21037/jtd-2019-rts-10

\section{Introduction}

Myasthenia gravis (MG) is a prototype autoimmune disease which usually presents with early fatigability, decreased motor neuron response with repetitive electrical stimulations, symptomatic improvement with drugs that cause inhibition of acetylcholine esterase, and the presence of acetylcholine receptor antibodies (AChR). Initially, Blalock (1,2), and later Keynes (3), described transsternal thymectomy in patients without a thymoma and recommended complete thymectomy for all patients with generalized MG. In the 1960's autoimmune nature of the disease was initially described $(4,5)$.

Keynes was the first to describe that MG patients with thymomas do not respond as well to thymectomy as those with just hyperplasia (3). Thereafter, Masaoka demonstrated improved outcomes of thymectomy in younger patients with MG (6). As a result, there is a consensus to treat MG patients with thymectomy, especially in younger patients, with no evidence of a thymoma. In patients with an elevated pre-operative AchR antibody titer, a decrease in AchR antibody titer post-operatively was a measurement of success. This was especially true in patients with noninvoluted hyperplastic thymus glands. 
Historically thymectomies were performed via sternotomy (1-3). Prior to 2002, thymectomy via partial upper sternal splitting was the senior author's preferred approach. The disadvantages of that approach included prolonged intubation with longer intensive care unit stay and hesitations on the part of the patients and the referring neurologist to accept thymectomy as a treatment option for MG.

In general, thymectomy is associated with improvement in MG symptoms in almost $75 \%$ of patients while the other $25 \%$ either showed no change or worsening symptoms. This improvement is more evident in following patient groups: younger age, nonthymomatous MG, positive AchR antibody and generalized MG.

\section{Understanding MG and reason of differences in the outcomes}

In $M G$ antibodies against the acetylcholine receptors (AChR) are present in $85 \%$ of patients (7) while Muscle Specific Kinase (MuSK) antibodies are present in another $6 \%$ (8). The remainder of the patients are negative for both acetylcholine receptor antibody (AChR) and MuSK antibodies, which are referred as "Double Seronegatives". More recently, a group of antibodies against low density lipoprotein receptor related protein 4 (LPR4) was discovered, which is most commonly seen in double seronegative patients (9-11). Thymic pathologies are more commonly seen in patients with AChR antibody positive patients than AChR or MuSK negative patients. Although AchR titers correlate poorly with disease severity but titers should be checked prior to initiating an immune modulating therapy as this may lead to seronegativity (12).

The patient selection for thymectomy among myasthenic patients is critical. The presence or absence of various antibodies and thymic pathology leads to variable outcomes after thymectomy. For example, Pensoti et al., in a single center nonrandomized study, compared post-thymectomy outcomes in patients with negative and positive serum AChR antibody. They reported better long-term outcomes in patients with positive serum AChR antibodies. They also noted that double sero-negative patients did worse than MuSK negative patients (13). Similarly, the underlying thymic pathology also plays an important role in patient selection for thymectomy. Thymectomy is associated with better outcomes in patients with thymic hyperplasia than other pathologies and the outcomes are even better if thymectomy is performed soon after the onset of myasthenia symptoms $(14,15)$. This is believed to be due to the removal of the thymus gland (AChR antibody source) leading to decrease serum level of antibodies hence improvement of symptoms.

The presence or absence of clinical symptoms like ptosis, diplopia, dyspnea, dysphagia, slurred speech and fatigue play an important role in planning of the timing of surgery. The patients should be evaluated by neurologist to determine if perioperative plasmapheresis or IVIG is required. Thymectomy should never be an emergency. In such symptomatic patients the surgery should be postponed till patients are optimized.

\section{Transsternal thymectomy: advantages and disadvantages}

Thymectomy via complete or partial sternotomy has been the gold standard till recently. This approach leads to optimum exposure of the whole mediastinum, phrenic nerves and other vital structures. Complete radical thymectomy is more reproducible via this approach. This approach is associated with low incidence of phrenic nerve or vascular injury (16). However, this approach has several reported disadvantages too. These include but are not limited to morbidity of sternotomy, longer operative time, extended postoperative hospitalization and prolonged recovery. This indeed was one of the main reasons for historically low appetite for thymectomy among both patients and the referring providers.

\section{Transcervical thymectomy}

This approach is considered to be the earliest example of minimally invasive technique for thymic surgery and was mostly done in young female patients. The advantages of this technique include cervical incision with less pain, shorter hospitalization and fewer complications (17). However, this approach is believed to be associated with suboptimal exposure and increase possibility of incomplete thymic resection.

\section{Video-assisted thoracic surgery (VATS) thymectomy}

This approach was popularized in early 2000s. It is associated with excellent exposure, acceptable operative time, low morbidity, short hospital stays and faster recovery. Most authorities who VATS thymectomy via bilateral approach, starting from left side and completing 


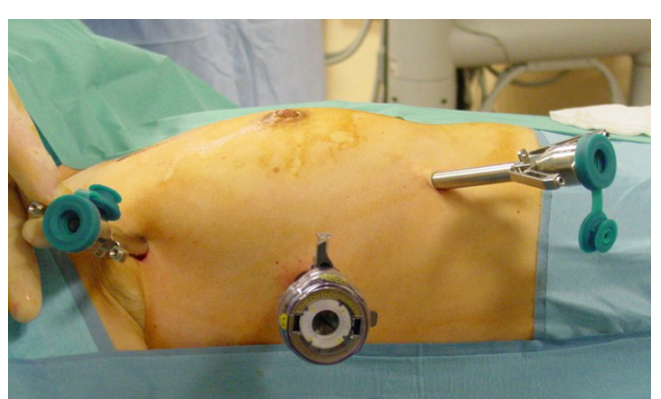

Figure 1 Port incisions for right sided Robotic thymectomy.

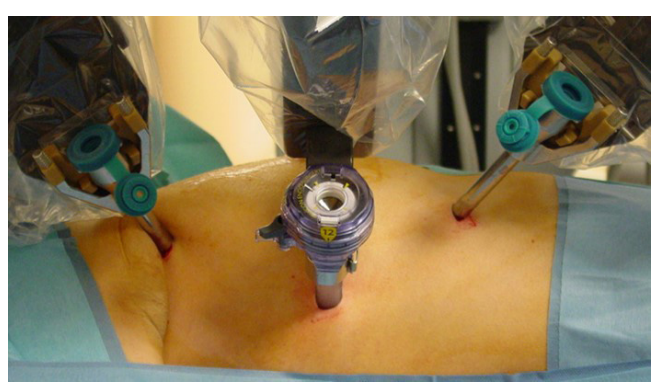

Figure 2 Docking for the right sided thymectomy.

via right VATS, perform a phrenic nerve to phrenic nerve thymectomy. Others have described either only left sided or right sided approach. In recent years the VATS subxiphoid approach has been popularized. Increasing reports of uniporter VATS subxiphoid approach shows comparable outcomes (18). The disadvantages are reported to be 2-dimensional view and the steep learning curve (19).

\section{Robotic thymectomy}

In recent years, robotic-assisted thoracic surgery (RATS) has emerged as a major alternative to both open or VATS approaches. There has been abundant literature published on robotic thymectomy for MG and thymomas during the last decade. Last decade has seen abundant literature. During the last decade there has been a plethora of lite. In the setting of MG and thymomas, resection of the thymus using robotic surgical techniques has been reported widely during the last decade. A detailed radiologic examination is necessary for proper candidate selection as well as determining the optimum surgical approach. Additionally, patients with MG are required to be approved for surgery by a qualified neurologist and anesthesiologist.

In this presentation, we present thymectomy technique (da Vinci Systems Intuitive Surgical, Sunnyvale, California,
USA) in West Virginia University, Heart and Vascular Institute and outcomes from the literature.

\section{Technical details}

\section{Preparation of surgery-positioning of the patient and docking of the robot DaVinci}

A double lumen endotracheal tube is placed with the help of a bronchoscope. A right sided approach is preferred because of the larger working space in the right hemithorax as compared to left, providing ample space for ports. The patient is placed in a 30 -degree semi-supine position. A roll is placed under the right shoulder, and the right arm is draped and positioned inferiorly near the chest. The table is then rotated towards surgeon.

We use three ports in this surgery. We do not use an assistant port. The incisions are done around the breast without violating the mammarian tissue (Figure 1). The first port placed is the camera port just lateral to the breast and a 30-degree up camera is used while placing the other ports. During the operation, 30 degrees camera either up or down could be used. The left port is placed in the anterior axillary fossa inside the pectoralis major muscle and the right port is placed in the 5-6th intercostal space right under the breast folds. An open access port is only required for a thymoma resection. For thymoma resections, the left or right port is enlarged and an Alexis retractor (Applied Medical, Rancho Santa Margarita, CA, USA) is placed. This could be used as an assistant port as well. After the placement of the ports, side docking of the robot is performed (Figure 2). All procedures performed in studies involving human participants were in accordance with the ethical standards of the institutional and/or national research committee(s) and with the Helsinki Declaration (as revised in 2013). Written informed consent was obtained from the patient.

\section{Surgical techniques}

\section{Right sided approach}

Carbon dioxide insufflation is used with a pressure of $8 \mathrm{mmHg}$ until the contralateral (left sided) mediastinal pleura is opened. A prograsper for the left arm and Maryland or bipolar forceps for the right arm is preferred. Begin by performing a thorough exploration of the cavity to identify of the borders of thymus and fatty tissue and its relationship with the phrenic nerve. Dissection begins with resection of the right sided pericardiophrenic fatty tissue. 


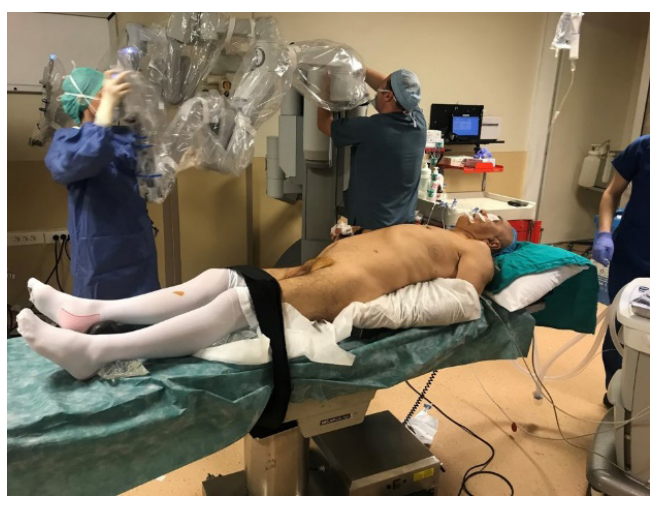

Figure 3 Positioning is same with the right sided robotic approach.

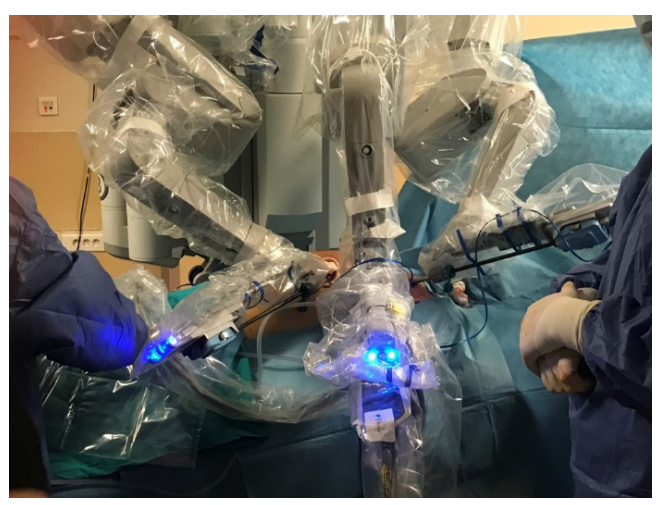

Figure 4 Docking is from the right side and anterior port is enlarged with a soft tissue retractor to provide help and remove the thymoma.

The resection of the thymus begins by dissecting the thymus on the pericardium anterior to the phrenic nerve with blunt dissection or Maryland or bipolar forceps in the right arm.

The thymus is separated from the sternum and the contralateral mediastinal pleura is opened. The dissection of the thymus from the pericardium around the superior vena cava, parallel to the phrenic nerve provides good exposure for visualization of junction of both innominate veins. Meticulous dissection and careful attention needed during this part to avoid vascular injury. The injury can occur during the separation of the upper poles and thyrothymic ligament from the left innominate vein. The dissection of poles is started by pulling down the right pole caudally and subsequently the left pole is dissected and pulled down. The attention is paid not to disrupt the thymic capsule during the dissection from the superior poles down to the thyrothymic ligament. Next is to identify and divide the venous branches to the thymus draining into the left innominate vein. The number of major thymic veins and their locations can vary between patients. Most patients have only one, but they can have up to four. Ligaclips or any energy device may be used to divide these vessels.

After complete dissection of the upper poles and division of thymic vein(s), the thymus is retracted caudally and is dissected off the innominate vein. The left side of the thymus can be dissected from the pericardium by pulling the thymus toward the surgeon. The left phrenic nerve can be visualized at this time by using a 30-degree down camera. After dissection of the left thymus from the pericardium, fatty tissue located at the left pericardiophrenic angle is resected.

The specimen is removed with an Endo bag (Covidien, USA) from the left upper port if an access port is not opened. The mediastinum is carefully inspected for any remaining fatty mediastinal tissue and hemostasis is obtained. One $10 \mathrm{~mm}$ Jackson Pratt drain is placed through the most anterior port across the mediastinum to drain both sides of the chest. This surgical technique is presented elsewhere in detail with a video presentation (20).

\section{Left sided approach}

Left sided surgery can be performed with both surgical robots SI and XI as presented in the right sided approach. Some prefer to use the XI because it consists of thinner and longer instruments, has the option to move the camera to any port, and the flexibility to approach the robot from any location. Because of these capabilities, surgeons who had previously preferred to perform a right sided thymectomy, are now using the left sided approach (21).

The most important maneuver while performing a left sided thymectomy is port placement. Inexperience and careless entry may cause cardiac injury. The positioning of the patient is almost symmetrical to the right side, except a roll is placed under the left side of the chest and the left arm is placed down on the arm board attached to the body (Figure 3). The first port is inserted carefully by avoiding any potential injuries to heart and lung. We place three ports around the mammary gland. A pressure of $7 \mathrm{mmHg}$ and Flow of $20 \mathrm{~L} / \mathrm{min}$ is recommended (21). We still prefer to use three ports without an assistant port (Figure 4). We prefer to start the dissection from the left upper pole and remove the fatty tissue and the thymus in the chest thereafter.

\section{Bilateral approach}

Robotic surgical technique for an extended thymectomy 


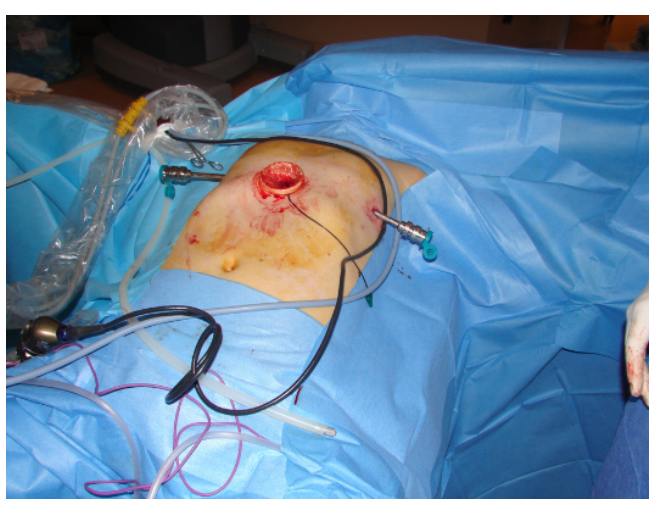

Figure 5 A wound retractor at the subxiphoid region, which is placed by a camera and 2 ports from each thoracic cavity are the incisions.

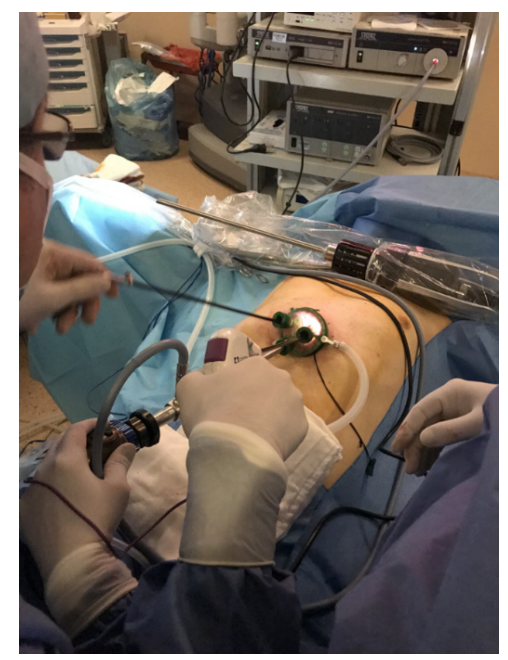

Figure 6 Dissection of fatty tissue and creation of a space is essential before docking.

have been recommended with bilateral surgeries (22). This technique involves placing the patient in the supine position, keeping the right arm below the body as recommended in right sided approach, and keeping the left arm elevated toward the head to secure a large area for left sided surgery. Right sided surgery was performed via 3 ports by docking from the left shoulder side. After dissection of the right sided thymus, pleura and fatty tissue, they then describe docking from the left side of the patient. In general, the authors recommend starting from fatty tissue in the right pericardiophrenic angle and move up to pull down the right horn of the thymus. The thymic vein is then is divided. Similarly, the left sided dissection is recommended.
However, this procedure is more time consuming for it requires the repositioning of the operation table and robotic cart.

\section{Subxiphoid approach}

The subxiphoid approach was derived from the video thoracoscopic systems (18) and popularized by Suda T (18). This surgery can be considered for large thymomas that have potential invasion to the pericardium. The authors approach to a subxiphoid thymectomy is different than previous descriptions of thymectomies. First, fatty tissue anterior and lateral to the heart should be dissected to provide a space with VATS and uniportal devices (Figure 5). Then after creation of a space, the dissection of the thymic and mediastinal tissue can begin (Figure 6). The docking of both the DaVinci Si and Xi differ when performing the subxiphoid approach when comparing right and left approaches. The Si needs to be docked from the cranial side of the patient, which may disturb anesthesia. The benefit of the $\mathrm{Xi}$, is that it can dock laterally as the arms are mounted on the boom. $\mathrm{CO}_{2}$ use is still needed. Suda et al., recommends use of single lumen tube for most cases but double lumen tube may be necessary for thymoma with pulmonary invasion.

\section{Outcomes after robotic thymectomy for MG}

The da Vinci surgical robotic systems provides a safer surgical technique for thymectomies by improving maneuverability for dissection around vascular (innominate vein, superior vena cava, and aorta) and nerve (phrenic nerve and laryngeal nerve) structures. The radicality of conventional video-assisted thoracoscopic thymectomy could increase by improving the quality of the dissection in difficult-to-reach areas such as the contralateral mediastinum and supra innominate vein region.

Ruckert and colleagues presented their outcomes after thoracoscopic and robotic thymectomy in MG patients. In patients who underwent robotic thymectomy, they claimed a statistically significant increase in complete stable remission rate (CSR) (39.2\% vs. 20.3\%) versus those who underwent thoracoscopic approach. They attributed this outcome to the radicality and the completeness of the resection (23).

Other authors confirmed better early outcomes such as low open conversion rate $(<5 \%)$ and morbidity $(<10 \%)$ rates as well as shorter hospital stays ( $<4$ days) with robotic thymectomy (24-26). A recent multi-institutional study 
demonstrated even better early outcomes such as no open conversion, $6 \%$ complication rate, no nervous or major vascular injuries, and a median hospital stay of 3 days (27).

MGFA describe CSRs ranging from 28\% to $42.8 \%$ for conventional thoracoscopic thymectomy $(28,29)$ and from $27 \%$ to $42 \%$ for robotic thymectomy $(24,30)$. Generally, there is a wide margin of discrepancy between the reported complete stable remission series. This variability may be due to various factors. These include the followings: different follow-up durations (18-45 months), heterogeneity of patient characteristics (thymomatous and nonthymomatous, ocular and general MG with different severity, variable length of preoperative symptoms), clinical evaluations failed to be performed by a neurologist, different level of immunosuppressive use, different time lapse after the ingestion of pyridostigmine and physical examination, different prescriptions and different management strategy for weaning off drugs after thymectomy.

Uncertainties remain concerning the role of thymectomy for patients with purely ocular symptoms, seronegative disease, late-onset disease, and involvement of ectopic thymic tissue.

Between $30 \%$ and $70 \%$ of patients who initially only experience ocular symptoms will eventually develop generalized myasthenia. Thus, some authors (31) have advocated surgery for patients with purely ocular MG. Our practice is to include these patients, particularly when the symptoms are disabling and poorly controlled by medical therapy. Some authors demonstrated a higher CSR rate and improvement in patients with ocular and mild MG. A classic article, published by the American Academy of Neurology, demonstrated better clinical outcomes after thymectomy with severe MG (31).

The indication for thymectomy in AchR antibody negative patients is still controversial. This is a heterogeneous group with false negative results. A retrospective study demonstrated similar outcomes in AchR ab negative and AchR antibody positive patients (32). It is well known that the presence of MuSK antibodies is a predictor of poor outcomes (33). Data suggests that a thymectomy should not be performed in MuSK antibodypositive patients. All seronegative patients should be tested for the presence of MuSK antibodies, and only double seronegative patients could be considered eligible for surgery. In contrary some studies some studies have shown slightly higher CSR in seronegative patients when compared to the Ab AchR-positive group (34). In the post thymectomy non-responder patients the presence of ectopic thymic tissue should be considered and may be present in up to $25 \%$.

\section{Conclusions}

Thymectomy continues to play an important role in the management of MG. The outcomes of thymectomy in MG patients are comparable among all approaches. Robotic platform, due to its enhanced visibility and articulating instrument, does provide a unique opportunity to perform radical thymectomy through either left or right chest or via subxiphoid approach with minimal morbidity and good long-term outcomes.

\section{Acknowledgments}

Funding: None.

\section{Footnote}

Provenance and Peer Review: This article was commissioned by the editorial office, Journal of Thoracic Disease for the series "Robotic Thoracic Surgery". The article has undergone external peer review.

Conflicts of Interest: All authors have completed the ICMJE uniform disclosure form (available at: http://dx.doi. org/10.21037/jtd-2019-rts-10). The series "Robotic Thoracic Surgery" was commissioned by the editorial office without any funding or sponsorship. GA served as the Guest Editor of the series and serves as an unpaid editorial board member of Fournal of Thoracic Disease. The authors have no other conflicts of interest to declare.

Ethical Statement: The authors are accountable for all aspects of the work in ensuring that questions related to the accuracy or integrity of any part of the work are appropriately investigated and resolved. All procedures performed in studies involving human participants were in accordance with the ethical standards of the institutional and/or national research committee(s) and with the Helsinki Declaration (as revised in 2013). Written informed consent was obtained from the patient.

Open Access Statement: This is an Open Access article distributed in accordance with the Creative Commons Attribution-NonCommercial-NoDerivs 4.0 International License (CC BY-NC-ND 4.0), which permits the non- 
commercial replication and distribution of the article with the strict proviso that no changes or edits are made and the original work is properly cited (including links to both the formal publication through the relevant DOI and the license). See: https://creativecommons.org/licenses/by-nc-nd/4.0/.

\section{References}

1. Blalock A. Thymectomy in the treatment of Myasthenia gravis. Report of 20 cases. J Thorac Surg 1944;13:316-39.

2. Blalock A, Harvey MA, Ford Fr, et al. The treatment of myasthenia gravis by removal of thymus gland. JAMA 1941;117:1529-33.

3. Keynes G. Surgery for thymus gland. Br J Surg 1946;33:201-14.

4. Simpson JA. Myasthenia Gravis. A new hypothesis. Scot Med J 1960;4:419-36.

5. Nastuk WL, Plescia OJ, Osserman KE. Changes in the serum complement activity in patients with myasthenia gravis. Proc Soc Exp Biol Med 1960;105:177-84.

6. Masaoka A, Yamakawa Y, Niwa H, et al. Extended thymectomy for myasthenia gravis patients: a 20-year review. Ann Thorac Surg 1996;62:853-9.

7. Meriggioli MN, Sanders DB. Autoimmune myasthenia gravis:emerging clinical and biological heterogeneity. Lancet Neurol 2009;8:475-90.

8. Hoch W, McConville J, Helms S, et al. Autoantibodies to the receptor tyrosine kinase MuSK in patients with myasthenia gravis without acetylcholine receptor antibodies. Nat Med 2001;7:365-8.

9. Leite MI, Jacob S, Viegas S, et al. IgG1 antibodies to acetylcholine receptors in seronegative myasthenia gravis. Brain 2008;131:1940-52.

10. Higuchi O, Hamuro J, Motomura M, et al. Autoantibodies to lowdensity lipoprotein receptor related protein 4 in öyasthenia gravis. Ann Neurol 2011;69:418-22.

11. Zhang B, Tzartos JS, Belimezi M, et al. Autoantibodies to lipoprotein -related protein 4 in patients with myasthenia gravis. Arch Neurol 2012;69:445-51.

12. Chan KH, Lachance DH, Harper CM, et al. Frequency of seronegativity in adult-acquired generalized myasthenia gravis. Muscle Nerve 2007;36:651.

13. Ponseti JM, Caritg N, Gamez J, et al. A comparison of long term postthymectomy outcome of anti AChR positive and anti AChR negative and Anti MuSK positive patients with nonthymomatous myasthenia gravis. Expert Opin Biol Ther 2009;9:1-8.

14. Budde JM, Morris CD, Gal AA, et al. Predictors of outcome in thymectomy for myasthenia gravis. Ann Thorac Surg 2001;72:197-202.

15. Nieto IP, Robledo JP, Pajuelo MC, et al. Prognostic factors for myasthenia gravis treated with thymectomy: A review of 61 cases. Ann Thorac Surg 1999;67:1568-71.

16. Jaretzki A 3rd, Wolff M. "Maximal" thymectomy for myasthenia gravis. Surgical anatomy and operative technique. J Thorac Cardiovasc Surg 1988;96:711-6.

17. Calhoun RF, Ritter JH, Guthrie TJ, et al. Results of transcervical thymectomy for myasthenia gravis in 100 consecutive patients. Ann Surg 1999;230:555-9; discussion 559-61.

18. Suda T. Robotic Subxiphoid thymectomy. J Vis Surg 2016;2:118-23.

19. Mineo TC, Pompeo E, Lerut TE, et al. Thoracoscopic thymectomy in autoimmune myasthesia: results of leftsided approach. Ann Thorac Surg 2000;69:1537-41.

20. Kaba E, Cosgun T, Ayalp K, et al. Robotic thymectomy for myasthenia gravis. Ann Cardiothorac Surg 2019;8:288-91.

21. Ferrari-Light Dana, Cerfolio RJ. Left sided approach for robotic thymectomy: technical tips, advantages and drawbacks. Shanghai Chest 2019;3:2-7.

22. Kawaguchi K, Fukui T, Nakamura S, et al. A bilateral approach to extended thymectomy using Da Vinci surgical system for patients with myasthenia gravis. Surg Today 2018;48:195-9.

23. Rückert JC, Swierzy M, Ismail M. Comparison of robotic and nonrobotic thoracoscopic thymectomy: a cohort study. J Thorac Cardiovasc Surg 2011;141:673-7.

24. Rückert JC, Ismail M, Swierzy M, et al. Thoracoscopic thymectomy with the da Vinci robotic system for myasthenia gravis. Ann N Y Acad Sci 2008;1132:329-35.

25. Rea F, Marulli G, Bortolotti L, et al. Experience with the "da Vinci" robotic system for thymectomy in patients with myasthenia gravis: report of 33 cases. Ann Thorac Surg 2006;81:455-9.

26. Goldstein SD, Yang SC. Assessment of robotic thymectomy using the MyastheniGravis Foundation of America Guidelines. Ann Thorac Surg 2010;89:1080-5.

27. Marulli G, Schiavon M, Perissinotto E, et al. Surgical and neurologic outcomes after robotic thymectomy in 100 consecutive patients with myasthenia gravis. J Thorac Cardiovasc Surg 2013;145:730-5.

28. Keating CP, Kong YX, Tay V, et al. VATS thymectomy for nonthymomatous myasthenia gravis: standardized outcome assessment using the myasthenia gravis foundation of America clinical classification. Innovations (Phila) 2011;6:104-9. 
29. Tomulescu V, Ion V, Kosa A, et al. Thoracoscopic thymectomy mid-term results. Ann Thorac Surg 2006;82:1003-7.

30. Freeman RK, Ascioti AJ, Van Woerkom JM, et al. Longterm follow-up after robotic thymectomy for nonthymomatous myasthenia gravis. Ann Thorac Surg 2011;92:1018-22.

31. Gronseth GS, Barohn RJ. Practice parameter: thymectomy for autoimmune myasthenia gravis (an evidence-based review): report of the Quality Standards Subcommittee of the American Academy of Neurology. Neurology 2000;55:7-15.

32. Guillermo GR, Téllez-Zenteno JF, Weder-Cisneros N,

Cite this article as: Raza B, Dhamija A, Abbas G, Toker A. Robotic thymectomy for myasthenia gravis surgical techniques and outcomes. J Thorac Dis 2021;13(10):6187-6194. doi: 10.21037/ jtd-2019-rts-10 et al. Response of thymectomy: clinical and pathological characteristics among seronegative and seropositive myasthenia gravis patients. Acta Neurol Scand 2004;109:217-21.

33. Pompeo E, Tacconi F, Massa R, et al. Long-term outcome of thoracoscopic extended thymectomy for nonthymomatous myasthenia gravis. Eur J Cardiothorac Surg 2009;36:164-9.

34. Ponseti JM, Gamez J, Vilallonga R, et al. Influence of ectopic thymic tissue on clinical outcome following extended thymectomy in generalized seropositive nonthymomatous myasthenia gravis. Eur J Cardiothorac Surg 2008;34:1062-7. 